\title{
ON THE STRUCTURE OF LIPSCHITZ-FREE SPACES
}

\author{
MAREK CÚTH, MICHAL DOUCHA, AND PRZEMYSŁAW WOJTASZCZYK
}

\begin{abstract}
In this note we study the structure of Lipschitz-free Banach spaces. We show that every Lipschitz-free Banach space over an infinite metric space contains a complemented copy of $\ell_{1}$. This result has many consequences for the structure of Lipschitz-free Banach spaces. Moreover, we give an example of a countable compact metric space $K$ such that $\mathcal{F}(K)$ is not isomorphic to a subspace of $L_{1}$ and we show that whenever $M$ is a subset of $\mathbb{R}^{n}$, then $\mathcal{F}(M)$ is weakly sequentially complete; in particular, $c_{0}$ does not embed into $\mathcal{F}(M)$.
\end{abstract}

\section{INTRODUCTION}

Given a metric space $M$, it is possible to construct a Banach space $\mathcal{F}(M)$ in such a way that the Lipschitz structure of $M$ corresponds to the linear structure of $\mathcal{F}(M)$. This space $\mathcal{F}(M)$ is sometimes called "Lipschitz-free space". We refer to the next section for some more details concerning the construction and basic properties of those spaces. Although Lipschitz-free spaces over separable metric spaces are easy to define, their structure is poorly understood to this day. The study of the linear structure of Lipschitz-free spaces over metric spaces has become an active field of study, see e.g. [8, 9, 11, 13, 14, 18, 20]. In the first part of this paper we prove the following general result.

Theorem 1. Let $M$ be an infinite metric space. For the Banach space $X=\mathcal{F}(M)$, we have

(i) $\ell_{1} \stackrel{c}{\hookrightarrow} X$, i.e., there is a complemented subspace of $X$ isomorphic to $\ell_{1}$.

From this we get

(ii) $X \stackrel{\leftrightarrow}{\rightarrow} \mathcal{C}(K)$, i.e., $X$ is not isomorphic to a complemented subspace of a $\mathcal{C}(K)$ space.

(iii) $X^{*}$ is not weakly sequentially complete; in particular, $X$ is not isomorphic to $L^{1}$-predual.

(iv) $X$ is not isomorphic to the Gurariu space.

(v) $X$ is a projectively universal separable Banach space, i.e., for any separable Banach space $Y$ there exists a bounded linear operator from $X$ onto $Y$.

It often happens that the Lipschitz-free space over a "small enough" space is isomorphic to $\ell_{1}$. For example, if $M \subset \mathbb{R}$ is a set of measure zero or if $M$ is a separable ultrametric space, then $\mathcal{F}(M)$ is isomorphic to $\ell_{1}$, see [13] and [7]. By the result of A. Dalet [8], $\mathcal{F}(K)$ is a dual space with MAP whenever $K$ is a countable compact metric space. Hence, one could conjecture that in this case $\mathcal{F}(K)$ is isomorphic to $\ell_{1}$. We give an example which shows that this is not the case.

Theorem 2. There is a countable compact metric space $K$ such that $\mathcal{F}(K) \leftrightarrow L_{1}$, i.e., $\mathcal{F}(K)$ is not linearly isomorphic to a subspace of $L_{1}$. Moreover, $K$ is a convergent sequence, i.e., it has only one accumulation point.

If $M$ contains a bi-Lipschitz copy of $c_{0}$, then $\mathcal{F}(M)$ is an isomorphically universal separable Banach space; i.e., $\mathcal{F}(M)$ contains an isomorphic copy of every separable Banach space (for more details we refer to Section (4). Y. Dutrieux and V. Ferenczi in [9] asked for the converse. The answer to this question is in general negative, because it follows from the result of P. Kaufmann

2010 Mathematics Subject Classification. 46B03, 54E35.

Key words and phrases. Lipschitz-free space, isomorphically universal separable Banach space, embedding of $c_{0}$. 
[18, Corollary 3.3] that $\mathcal{F}\left(c_{0}\right)$ is isomorphic to $\mathcal{F}\left(B_{c_{0}}\right)$ (thus, it is a universal) and of course, since $B_{c_{0}}$ is bounded, $B_{c_{0}}$ does not contain a bi-Lipschitz copy of $c_{0}$. However, we can still ask the question in the setting of Banach spaces.

Question 1. Let $X$ be a Banach space. Is $\mathcal{F}(X)$ universal if and only if $X$ contains a bi-Lipschitz copy of $c_{0}$ ?

The following result is a partial progress towards the answer to this question. Up to our knowledge, Question 1 is left open.

Theorem 3. Let $M \subset \mathbb{R}^{n}$ be an arbitrary set. Then $\mathcal{F}(M)$ is weakly sequentially complete.

Consequently, $c_{0} \leftrightarrow \mathcal{F}(M)$; i.e., $c_{0}$ is not linearly isomorphic to a subspace of $\mathcal{F}(M)$.

To the best of our knowledge, it was not even known whether there could be a Lipschitz-free space which neither embeds into $L_{1}$ nor is universal. The example given in Theorem 2 is one such example (because, by the result of A. Dalet [8], $\mathcal{F}(K)$ is a separable dual space and so it does not contain $\left.c_{0}\right)$. Another one is the space $\mathcal{F}\left([0,1]^{n}\right)$, see Theorems 3 and 7 .

In the last section of this note we mention some open problems related to the structure of isomorphically universal Lispchitz-free Banach spaces.

The notation and terminology we use are relatively standard. If $X$ and $Y$ are Banach spaces, the symbol $Y \hookrightarrow X$ (resp. $Y \hookrightarrow X$ ) means that $Y$ is (resp. is not) linearly isomorphic to a subspace of $X$. If $(M, d)$ is a metric space, $x \in M$ and $r \geq 0$, we use $U(x, r)$ and $B(x, r)$ to denote respectively the open and closed ball, i.e., the set $\{y \in M: d(x, y)<r\}$ and $\{y \in M: d(x, y) \leq r\}$.

\section{BASIC FACTS ABOUT LIPSCHITZ-FREE SPACES}

Let $(M, d)$ be a metric space with a distinguished point denoted by 0 . Consider the space $\operatorname{Lip}_{0}(M)$ of all real-valued Lipschitz functions that map $0 \in M$ to $0 \in \mathbb{R}$. It has a vector space structure and one can define a norm $\|\cdot\|_{\text {Lip }}$ on $\operatorname{Lip}_{0}(M)$, where for $f \in \operatorname{Lip}_{0}(M),\|f\|_{\text {Lip }}$ is the minimal Lipschitz constant, i.e., $\sup \left\{\frac{|f(x)-f(y)|}{d(x, y)}: x \neq y \in M\right\}$. Then $\left(\operatorname{Lip}_{0}(M),\|\cdot\|_{\text {Lip }}\right)$ is a Banach space.

For any $x \in M$ denote by $\delta_{x} \in \operatorname{Lip}_{0}(M)^{*}$ the evaluation functional, i.e., $\delta_{x}(f)=f(x)$ for every $f \in \operatorname{Lip}_{0}(M)$. Denote by $\mathcal{F}(M)$ the closure of the linear span of $\left\{\delta_{x}: x \in M\right\}$ with the dual space norm denoted simply by $\|\cdot\|$. Observe that for any $x, y \in M$ we have $\left\|\delta_{x}-\delta_{y}\right\|=d(x, y)$.

This space is usually called Lipschitz-free Banach space (also Arens-Eells space) and it is uniquely characterized by the following universal property.

Let $X$ be a Banach space and suppose $L: M \rightarrow X$ is a Lipschitz map such that $L(0)=0$. Then there exists a unique linear map $\widehat{L}: \mathcal{F}(M) \rightarrow X$ extending $L$, i.e., the following diagram commutes

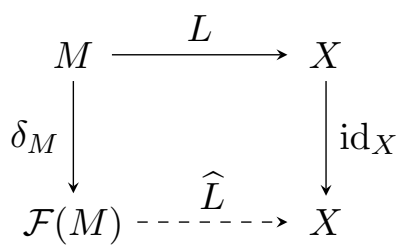

and $\|\widehat{L}\|=\|L\|_{\text {Lip }}$ where $\|\cdot\|_{\text {Lip }}$ denotes the Lipschitz norm of $L$.

This fact is usually referred to as folklore. The proof is so simple that we include it here.

Fix a Banach space $X$ and a Lipschitz map $L: M \rightarrow X$ mapping 0 to 0 . Extend linearly $L$ from $M$ onto $\operatorname{span}\left\{\delta_{x}: x \in M\right\}$ and denote this extension by $\widehat{L}$. We only need to check that $\|\widehat{L}\|_{\text {Lip }}=\|L\|_{\text {Lip }}$. Pick some $a \in \operatorname{span}\left\{\delta_{x}: x \in M\right\}$. Then $\|\widehat{L}(a)\|_{X}=f(\widehat{L}(a))$ for some 
$f \in B_{X^{*}}$. However, $f \circ L$ then belongs to $\operatorname{Lip}_{0}(M)$ and $\|f \circ L\|_{\text {Lip }} \leq\|L\|_{\text {Lip. }}$. It follows that $\|a\|\|L\|_{\text {Lip }} \geq\|\widehat{L}(a)\|_{X}$ which proves the claim. Then we can extend $\widehat{L}$ to $\mathcal{F}(M)$, the closure of $\operatorname{span}\left\{\delta_{x}: x \in M\right\}$.

Using this universal property of $\mathcal{F}(M)$, it is immediate that $\mathcal{F}(M)^{*}=\operatorname{Lip}_{0}(M)$. Indeed, it is enough to consider $X=\mathbb{R}$ in the universal property mentioned above.

Further, it is useful to observe that whenever $N$ is a subspace of a metric space $(M, d)$, then $\mathcal{F}(N)$ is linearly isometric to a subspace of $\mathcal{F}(M)$. Indeed, the isometry is determined by sending $\delta_{x} \in \mathcal{F}(N)$ to $\delta_{x} \in \mathcal{F}(M)$; in order to see it is an isometry it is enough to use the well-known fact that any $f \in \operatorname{Lip}_{0}(N)$ can be extended to $F \in \operatorname{Lip}_{0}(M)$ with $\|f\|_{\text {Lip }}=\|F\|_{\text {Lip }}$, e.g., by putting $F(x):=\inf \left\{f(n)+\|f\|_{\text {Lip }} d(n, x): n \in N\right\}, x \in M$; see e.g. [16, Lemma 7.39]. Using this observation together with the universal property of $\mathcal{F}(M)$ we see that the Lipschitz structure of $M$ corresponds to the linear structure of $\mathcal{F}(M)$. For example, if $N$ is bi-Lipschitz equivalent (resp. isometric) to a subset of $M$, then $\mathcal{F}(N)$ is linearly isomorphic (resp. linearly isometric) to a subspace of $\mathcal{F}(M)$, etc.

The last basic fact we would like to mention here is that it is possible to give an 'internal' definition of the norm on $\mathcal{F}(M)$, i.e. by a formula which refers only to the metric on the metric space $M$. This is in contrast to the 'external' definition given above which refers to the space $\operatorname{Lip}_{0}(M)$ in the computation of the norm. This is described e.g. in [27]. The proof is not difficult and so we include it here as well.

Let us consider another norm, denoted by $\|\cdot\|_{K R}$, on $\operatorname{span}\left\{\delta_{x}: x \in M\right\}$ which is a variant of the so-called Kantorovich-Rubinstein metric, a concept that penetrated many areas of mathematics and computer science. Let us identify $\delta_{0}$ with $0 \in \mathcal{F}(M)$. For $a \in \operatorname{span}\left\{\delta_{x}: x \in M \backslash\{0\}\right\}$ set

$$
\|a\|_{K R}=\inf \left\{\left|\alpha_{1}\right| \cdot d\left(y_{1}, z_{1}\right)+\ldots+\left|\alpha_{n}\right| \cdot d\left(y_{n}, z_{n}\right): a=\alpha_{1}\left(\delta_{y_{1}}-\delta_{z_{1}}\right)+\ldots+\alpha_{n}\left(\delta_{y_{n}}-\delta_{z_{n}}\right)\right\} .
$$

It is straightforward to check that $\|\cdot\|_{K R}$ is a seminorm. Moreover, it is the largest seminorm $\|\cdot\|^{\prime}$ on $\operatorname{span}\left\{\delta_{x}: x \in M\right\}$ satisfying $\left\|\delta_{x}-\delta_{y}\right\|^{\prime} \leq d(x, y)$ for every $x, y \in M$. Indeed, any seminorm $\|\cdot\|^{\prime}$ with that property must satisfy the inequality $\|x\|^{\prime} \leq\left|\alpha_{1}\right|\left\|\delta_{y_{1}}-\delta_{z_{1}}\right\|^{\prime}+\ldots+\left|\alpha_{n}\right|\left\|\delta_{y_{n}}-\delta_{z_{n}}\right\|^{\prime}$ when $x=\alpha_{1}\left(\delta_{y_{1}}-\delta_{z_{1}}\right)+\ldots+\alpha_{n}\left(\delta_{y_{n}}-\delta_{z_{n}}\right)$ which shows that $\|x\|^{\prime} \leq\|x\|_{K R}$. Since the standard norm $\|\cdot\|$ on $\mathcal{F}(M)$ satisfies the condition, we get that $\|\cdot\| \leq\|\cdot\|_{K R}$ which implies that $\|\cdot\|_{K R}$ is actually a norm and that $\left\|\delta_{x}-\delta_{y}\right\|_{K R}=d(x, y)$ for every $x, y \in M$.

Consider now the identity mapping $L: M \rightarrow \overline{\operatorname{span}\left\{\delta_{x}: x \in M\right\}} \|^{\|\cdot\|_{K R}}$ sending $x$ to $\delta_{x}$. It is an isometric embedding. By the universality property of $\mathcal{F}(M), L$ extends to $\widehat{L}: \mathcal{F}(M) \rightarrow$ $\overline{\operatorname{span}\left\{\delta_{x}: x \in M\right\}}{ }^{\|\cdot\|_{K R}}$ which is still 1-Lipschitz. It follows that $\|\cdot\|_{K R} \leq\|\cdot\|$, so the norms $\|\cdot\|$ and $\|\cdot\|_{K R}$ are one and the same. This fact is often referred to as the Kantorovich duality.

\section{Embedding of $\ell_{1}$}

The purpose of this section is to prove Theorem 1. It will be deduced from the fact that $\ell_{\infty}$ embeds in the dual of a Lipschitz-free space, i.e., into the space of Lipschitz functions. Let us note that we do not know whether $\ell_{\infty}$ embeds isometrically into $\operatorname{Lip}_{0}(M)$ for every infinite metric space $M$. The natural way of embedding $\ell_{\infty}$ into the space of Lipschitz functions is described in the Lemma below.

Lemma 4. Let $(M, d)$ be a metric space, $K>0$ and let $\left(x_{n}, y_{n}\right)_{n \in \mathbb{N}}$ be a sequence of pairs of points from $M$ satisfying the following three conditions.

(i) For every $n \in \mathbb{N}$, we have $x_{n} \neq y_{n}$.

(ii) For every $n, m \in \mathbb{N}$, we have $x_{m} \notin U\left(y_{n}, K \cdot d\left(y_{n}, x_{n}\right)\right)$.

(iii) For every $n \neq m$, we have $U\left(y_{n}, K \cdot d\left(y_{n}, x_{n}\right)\right) \cap U\left(y_{m}, K \cdot d\left(y_{m}, x_{m}\right)\right)=\emptyset$.

Then $\ell_{\infty} \hookrightarrow \operatorname{Lip}_{0}(M)$. 
Proof. We may without loss of generality assume that $0=x_{1}$ (because $\operatorname{Lip}_{0}(M) \ni f \mapsto f-f\left(x_{1}\right)$ is a linear isometry onto the space of Lipschitz functions $g$ with $\left.g\left(x_{1}\right)=0\right)$. For every $n \in \mathbb{N}$ we define $f_{n}(x):=\max \left\{d\left(y_{n}, x_{n}\right)-\frac{d\left(y_{n}, x\right)}{K}, 0\right\}, x \in M$. Then $f_{n} \in \operatorname{Lip}(M)$. Moreover, it is easy to see that $\left\|f_{n}\right\|_{\text {Lip }} \leq \frac{1}{K}$ and $\|f\|_{\infty}=d\left(y_{n}, x_{n}\right)$. By (iii), we have $K \cdot d\left(x_{n}, y_{n}\right) \leq d\left(x_{1}, y_{n}\right)$; hence, $f_{n}(0)=0$.

Notice that condition (iii) implies that if $f_{n}(x) \neq 0$, then for every $m \neq n$ we have $f_{m}(x)=0$. For every $x \in M$, we denote by $n(x)$ the unique $n \in \mathbb{N}$ with $f_{n}(x) \neq 0$ if it exists; otherwise, we put $n(x):=1$. Finally, we define $T: \ell_{\infty} \rightarrow \operatorname{Lip}_{0}(M)$ by

$$
T(\alpha)(x):=\alpha(n(x)) \cdot f_{n(x)}(x), \quad \alpha=(\alpha(n))_{n \in \mathbb{N}} \in \ell_{\infty} .
$$

First, we will show that $T$ is linear and $\|T\| \leq \frac{2}{K}$. It is easy to see that $T$ is linear; hence, it suffices to show that for $\alpha=(\alpha(n))_{n \in \mathbb{N}} \in \ell_{\infty}$ with $\|\alpha\|=1$, we have $\|T(\alpha)\|_{\text {Lip }} \leq \frac{2}{K}$. Fix $x, y \in M$. We need to show that $|T(\alpha)(x)-T(\alpha)(y)| \leq \frac{2}{K} d(x, y)$. If $n(x)=n(y)$, this is easy because $f_{n(x)}$ is $\frac{1}{K}$-Lipschitz. Hence, we may assume that $n(y) \neq n(x)$. Thus, we have $f_{n(x)}(y)=0=f_{n(y)}(x)$ and $|T(\alpha)(x)-T(\alpha)(y)| \leq f_{n(x)}(x)+f_{n(y)}(y)=\left|f_{n(x)}(x)-f_{n(x)}(y)\right|+\left|f_{n(y)}(y)-f_{n(y)}(x)\right| \leq \frac{2}{K} d(x, y)$.

In order to see that $T$ is an isomorphism, we will use condition (iii). Fix $\alpha=(\alpha(n))_{n \in \mathbb{N}} \in \ell_{\infty}$ and $N \in \mathbb{N}$. By (iii), for every $k \in \mathbb{N}$, we have $f_{k}\left(x_{N}\right)=0$; hence $T(\alpha)\left(x_{N}\right)=0$ and we have

$$
\left|T(\alpha)\left(x_{N}\right)-T(\alpha)\left(y_{N}\right)\right|=\left|T(\alpha)\left(y_{N}\right)\right|=|\alpha(N)| f_{N}\left(y_{N}\right)=|\alpha(N)| d\left(x_{N}, y_{N}\right) .
$$

Therefore, $\|T(\alpha)\|_{\text {Lip }} \geq|\alpha(N)|$ and, since $N$ was arbitrary, $\|T(\alpha)\|_{\text {Lip }} \geq\|\alpha\|_{\infty}$.

The following result is the main step towards the proof of Theorem 1.

Theorem 5. Let $M$ be an infinite metric space. Then $\ell_{\infty} \hookrightarrow \operatorname{Lip}_{0}(M)$.

Proof. First, note that we may without generality assume that $M$ is complete, because otherwise we take the completion $N$ of $M$ and use the obvious fact that $\operatorname{Lip}_{0}(\bar{A})$ is linearly isometric to $\operatorname{Lip}_{0}(A)$ for every $A \subset N$; in particular, $\operatorname{Lip}_{0}(N)$ is linearly isometric to $\operatorname{Lip}_{0}(M)$.

Now, we will prove the statement considering several cases. In each of them we will find a sequence of pairs of points from $M$ satisfying the assumptions of Lemma 4 .

Case 1. $M$ is unbounded; i.e., for every $K>0$ there are $x, y \in M$ with $d(x, y)>K$.

Proof for Case 1. Pick a sequence $\left(z_{n}\right)_{n=1}^{\infty}$ in $M$ such that, for every $n \in \mathbb{N}$ we have $d\left(z_{n+1}, 0\right)>$ $2 d\left(z_{n}, 0\right)$. Now, for each $n \in \mathbb{N}$, put $x_{n}:=z_{2 n-1}$ and $y_{n}:=z_{2 n}$. We will show that the sequence $\left(x_{n}, y_{n}\right)_{n \in \mathbb{N}}$ satisfies the assumptions of Lemma 4 with $K=\frac{1}{3}$.

Obviously, (i) is satisfied. Further, for $n<m$ we have

$$
\begin{aligned}
& d\left(z_{n}, z_{m}\right) \leq d\left(z_{n}, 0\right)+d\left(z_{m}, 0\right)<\left(1+2^{-(m-n)}\right) d\left(z_{m}, 0\right), \\
& d\left(z_{n}, z_{m}\right) \geq d\left(z_{m}, 0\right)-d\left(z_{n}, 0\right)>\left(1-2^{-(m-n)}\right) d\left(z_{m}, 0\right) .
\end{aligned}
$$

Let us show that (ii) holds. We need to show that, for $n, m \in \mathbb{N}$, we have

$$
d\left(z_{2 m-1}, z_{2 n}\right) \geq \frac{1}{3} \cdot d\left(z_{2 n-1}, z_{2 n}\right) .
$$

This is obvious if $m=n$. If $m<n$, then we have

$$
d\left(z_{2 m-1}, z_{2 n}\right) \stackrel{(2)}{>}\left(1-2^{-(2 n-2 m+1)}\right) d\left(z_{2 n}, 0\right) \stackrel{(1)}{>}\left(1-2^{-3}\right)\left(1+2^{-1}\right)^{-1} d\left(z_{2 n}, z_{2 n-1}\right) .
$$

If $n<m$, then we have

$$
d\left(z_{2 m-1}, z_{2 n}\right) \stackrel{(2)}{>}\left(1-2^{-1}\right) d\left(z_{2 m-1}, 0\right)>\left(1-2^{-1}\right) d\left(z_{2 n}, 0\right) \stackrel{(1)}{>}\left(1-2^{-1}\right)\left(1+2^{-1}\right)^{-1} d\left(z_{2 n}, z_{2 n-1}\right) .
$$

This proves (3); hence, condition (ii) from Lemma 4 is satisfied. 
Finally, in order to see that (iii) holds, it is sufficient to see that, for $n \neq m$, we have

$$
d\left(z_{2 m}, z_{2 n}\right)>\frac{1}{3} \cdot\left(d\left(z_{2 n-1}, z_{2 n}\right)+d\left(z_{2 m-1}, z_{2 m}\right)\right) .
$$

We may assume that $n<m$ and then we have

$$
\begin{aligned}
d\left(z_{2 n-1}, z_{2 n}\right)+d\left(z_{2 m-1}, z_{2 m}\right) & \stackrel{\text { (1) }}{<}\left(1+2^{-1}\right)^{2} d\left(z_{2 m}, 0\right) \stackrel{\sqrt{2 n}}{<}\left(1+2^{-1}\right)^{2}\left(1-2^{-(2 m-2 n)}\right)^{-1} d\left(z_{2 m}, z_{2 n}\right) \\
& \leq\left(1+2^{-1}\right)^{2}\left(1-2^{-2}\right)^{-1} d\left(z_{2 m}, z_{2 n}\right)=3 d\left(z_{2 m}, z_{2 n}\right) .
\end{aligned}
$$

This proves (4); hence, condition (iii) from Lemma 4 is satisfied.

Case 2. $M$ is bounded and there is a closed infinite subset $N \subset M$ such that each point $n \in N$ is isolated in $N$.

Proof for Case 2. Fix $N$ as above. Since $M$ is bounded, there is $D>0$ such that, for every $x, y \in M$, we have $d(x, y) \leq D$. Since $N$ does not contain any nontrivial Cauchy sequence, there is an infinite subset $P \subset N$ which is uniformly discrete; i.e., there is $C>0$ such that, for every $x, y \in P$ with $x \neq y$, we have $d(x, y) \geq C$ (this is a simple exercise using the classical Ramsey theorem, see e.g. [24, Excercise 5.5]). Fix a one-to-one sequence $\left(a_{n}\right)_{n=0}^{\infty}$ of points from $P$ and, for every $n \in \mathbb{N}$, put $y_{n}=a_{n}$ and $x_{n}=a_{0}$. It remains to verify that the sequence $\left(x_{n}, y_{n}\right)_{n \in \mathbb{N}}$ satisfies the assumptions of Lemma 4 with $K=\min \left\{\frac{C}{2 D}, 1\right\}$. It is clear that conditions (ii) and (iii) are satisfied, because $K \leq 1$. Moreover, for every $n \in \mathbb{N}$, we have $K \cdot d\left(x_{n}, y_{n}\right) \leq K D \leq C / 2$; hence, $U\left(y_{n}, K \cdot d\left(x_{n}, y_{n}\right)\right) \subset U\left(y_{n}, C / 2\right)$ and, since $\left(y_{n}\right)_{n \in \mathbb{N}}$ is $C$-discrete, the balls are pairwise disjoint. This verifies condition (iii) from Lemma 4.

Case 3. $M$ is bounded and it contains infinitely many limit points.

Proof for Case 3. First, let us assume there is a sequence $\left(y_{n}\right)_{n \in \mathbb{N}}$ consisting of limit points in $M$ with $y_{n} \rightarrow y$. Then, for each $n \in \mathbb{N}$, put $r_{n}:=\operatorname{dist}\left(y_{n},\left\{y_{m}: m \neq n\right\}\right)>0$ and pick some $x_{n} \in U\left(y_{n}, r_{n} / 2\right)$ with $x_{n} \neq y_{n}$. Then it is easy to see that the sequence $\left(x_{n}, y_{n}\right)_{n \in \mathbb{N}}$ satisfies the assumptions of Lemma 4 with $K=1$. 2 ,

Otherwise, the set $N$ consisting of all the limit points in $M$ satisfies the assumptions of Case

Note that now it remains to handle the case when $M$ is compact and it contains a nontrivial convergent sequence consisting of isolated points. Indeed, by the already proven Cases 1-3, we may assume $M$ is bounded and contains only finitely many limit points. Then either $M$ is compact, or there is an infinite closed set of isolated points in $M$ and we may apply Case 2 .

Case 4. $M$ is compact and it contains a nontrivial convergent sequence consisting of isolated points.

Proof for Case 4. Let $\left(a_{n}\right)_{n \in \mathbb{N}}$ be a nontrivial convergent sequence consisting of isolated points with the limit point $a$. It is easy to construct by induction a sequence $\left(x_{n}, y_{n}\right)_{n \in \mathbb{N}}$ of pairs of points from $M$ with

(a) $\forall n \in \mathbb{N}: \quad y_{n} \in\left\{a_{n}: n \in \mathbb{N}\right\}$ and $y_{n} \notin\left\{y_{m}: m<n\right\} \cup\left\{x_{m}: m<n\right\}$,

(b) $\forall n \in \mathbb{N}: \quad d\left(y_{n}, a\right)<\min \left\{d\left(y_{n}, y_{m}\right): m<n\right\}$ and

(c) for every $n \in \mathbb{N}$, we pick $x_{n}$ to be any point with $d\left(x_{n}, y_{n}\right)=\operatorname{dist}\left(y_{n}, M \backslash\left\{y_{n}\right\}\right)$.

Now, having such a sequence $\left(x_{n}, y_{n}\right)_{n \in \mathbb{N}}$, it remains to check that it satisfies the assumptions of Lemma 4 with $K=1$. Obviously, (ii) is satisfied. Moreover, we have $d\left(x_{n}, y_{n}\right) \leq d\left(y_{n}, x\right)$ for every $x \in M \backslash\left\{y_{n}\right\}$ and so in order to verify (iii), it is enough to observe that, for $n, m \in \mathbb{N}$, we have $x_{n} \neq y_{m}$. This follows from (a) for $n<m$, from (c) for $n=m$ and from (b) for $n>m$, because in the last case we have $d\left(x_{n}, y_{n}\right) \leq d\left(y_{n}, a\right)<d\left(y_{n}, y_{m}\right)$.

It remains to verify (iii). But this is easy, because, for every $n \in \mathbb{N}$, by the choice of $x_{n}$ we have $U\left(y_{n}, d\left(x_{n}, y_{n}\right)\right)=\left\{y_{n}\right\}$. 

5 .

Since the cases mentioned above cover all the possibilities, this completes the proof of Theorem

Proof of Theorem 1. This is a consequence of Theorem 5. Indeed, it is a classical result that, for every Banach space $X, \ell_{\infty} \hookrightarrow X^{*}$ if and only if $\ell_{1}$ is isomorphic to a complemented subspace of $X$ [4, Theorem 4]; hence, (ii) follows. Since any complemented subspace of a $\mathcal{C}(K)$ space contains $c_{0}$, see e.g. [25, Theorem 5.1], from (ii) we get (iii) because $c_{0}$ is not isomorphic to a subspace of $\ell_{1}$. Since the dual space contains $c_{0}$, it is not weakly sequentially complete and so it is not isomorphic to $L^{1}(\mu)$ [28, Corollary III.C.14]. Therefore, $X$ is not isomorphic to any $L^{1}$-predual; in particular, not to the Gurariü space [15]; see also [12, Theorem 2.17]. As it is well known that $\ell_{1}$ is projectively universal, i.e., for any separable Banach space $Y$ there exists a bounded linear operator from $\ell_{1}$ onto $Y$, the same is true for $X$ since $\ell_{1}$ is complemented there.

Remark 6. From the assumptions of Lemma 4 it is possible not only to deduce that $\ell_{1}$ is isomorphic to a complemented subspace of $\mathcal{F}(M)$, but it is even possible to describe relatively easily this subspace. Let us assume that $\left(x_{n}, y_{n}\right)_{n \in \mathbb{N}}$ is as in Lemma 4. For each $n \in \mathbb{N}$, put $e_{n}:=\frac{\delta_{y_{n}}-\delta_{x_{n}}}{d\left(y_{n}, x_{n}\right)} \in$ $\mathcal{F}(M)$. Then, using similar proof as in Lemma 4 we get that $\left(e_{n}\right)_{n \in \mathbb{N}}$ is $2 / K$-equivalent to the $\ell_{1}$ basis. Moreover, consider functions $\left(f_{n}\right)_{n \in \mathbb{N}}$ from the proof of Lemma 4 and define $r: M \rightarrow \mathcal{F}(M)$ by $r(x):=\sum_{n \in \mathbb{N}} f_{n}(x) e_{n}, x \in M$. Then it is possible to verify that $r$ is a $2 / K$-Lipschitz. Using the universal property of $r$ we find $P: \mathcal{F}(M) \rightarrow \mathcal{F}(M)$ with $P \circ \delta=r$ and $\|P\| \leq 2 / K$. Finally, one can verify that $P$ is actually a projection onto $\overline{\operatorname{span}}\left\{e_{n}: n \in \mathbb{N}\right\}$.

\section{Embedding INTO $L_{1}$}

The purpose of this section is to prove Theorem 2. In order to prove it, we will need the following result. The proof is just a modification of the arguments from [19].

Theorem 7. For any measure $\mu, \mathcal{F}\left([0,1]^{2}\right) \hookrightarrow L_{1}(\mu)$.

Proof. In order to shorten our notation, put $I:=[0,1]^{2}$. If there is a measure $\mu$ with $\mathcal{F}(I) \hookrightarrow L_{1}(\mu)$, then there is a continuous linear mapping from $L_{\infty}(\mu)$ onto $\operatorname{Lip}_{0}(I)$. Since $L_{\infty}(\mu)$ is a commutative $\mathrm{C}^{*}$-algebra, there exists a compact Hausdorff space $K$ such that $L_{\infty}(\mu)$ is isometric to $\mathcal{C}(K)$. Hence, it suffices to show that there does not exist a bounded linear mapping $T: \mathcal{C}(K) \rightarrow \operatorname{Lip}_{0}(I)$ which is onto. We only show that the "identity" mapping $i d: \operatorname{Lip}_{0}(I) \rightarrow W^{1,1}(I)$ is absolutely summing. Then the rest can be proved just copying line by line the arguments from [19, Theorem 3], where this statement is proved for the space $\mathcal{C}^{1}(I)$ instead of $\operatorname{Lip}_{0}(I)$ using the fact that "identity" mapping $i d: \mathcal{C}^{1}(I) \rightarrow W^{1,1}(I)$ is "absolutely summing" ( $W^{1,1}(I)$ is the Sobolev space). So consider the "identity" mapping id $: \operatorname{Lip}_{0}(I) \rightarrow W^{1,1}(I)$. More precisely, having a Lipschitz function $f$, we denote by $[f]$ the equivalence class containing all the functions which are equal to $f$ almost everywhere. The "identity" mapping is the mapping $f \mapsto[f]$. By the classical Rademacher's theorem, see e.g. 23], every Lipschitz function defined on $I$ is almost everywhere differentiable and so it is possible to put $\|[f]\|_{W}:=\int_{[0,1]^{2}}\left(|f(x, y)|+\left|\partial_{1} f(x, y)\right|+\left|\partial_{2} f(x, y)\right|\right) \mathrm{d} x \mathrm{~d} y$. It is immediate that $\|[f]\|_{W} \leq 3\|f\|_{\text {Lip }}$ and it remains to show that the mapping $f \mapsto[f]$ is absolutely summing; i.e., there is a constant $C$ such that whenever $\left(f_{i}\right)_{i=1}^{m}$ are functions from $\operatorname{Lip}_{0}(I)$, then

$$
\sum_{i=1}^{m}\left\|\left[f_{i}\right]\right\|_{W} \leq C \sup \left\{\sum_{i=1}^{m}\left|x^{*}\left(f_{i}\right)\right|: x^{*} \in \operatorname{Lip}_{0}(I)^{*},\left\|x^{*}\right\| \leq 1\right\} \text {. }
$$

Let us define $\Phi: \operatorname{Lip}_{0}\left([0,1]^{2}\right) \rightarrow L_{\infty}(I) \oplus_{1} L_{\infty}(I) \oplus_{1} L_{\infty}(I)$ by $\operatorname{Lip}_{0}(I) \ni f \mapsto \Phi(f):=\left(f, \partial_{1} f, \partial_{2} f\right)$. Note that $\Phi$ is a linear bounded operator. Further, consider $\Psi: L_{\infty}(I) \oplus_{1} L_{\infty}(I) \oplus_{1} L_{\infty}(I) \rightarrow$ $L_{1}(I) \oplus_{1} L_{1}(I) \oplus_{1} L_{1}(I)$ defined as the identity. It is a standard fact, see e.g. [2, Remark 8.2.9], that the identity operator from $L_{\infty}(I)$ to $L_{1}(I)$ is absolutely summing; hence, $\Psi$ is absolutely summing. 
It is a classical fact that composition of a bounded operator with an absolutely summing one is absolutely summing, see e.g. [2, Proposition 8.2.5]. Hence, $i d=\Psi \circ \Phi$ is absolutely summing.

Remark 8. The result that $\mathcal{F}\left(\mathbb{R}^{2}\right) \hookrightarrow L_{1}$ is often mentioned as a result of A. Naor and G. Schechtmann [22]. The proof above shows that, using minor modifications, it actually follows already from [19].

The rest of this section is devoted to the proof of Theorem 2. First, we construct the countable compact space with one accumulation point and then in a series of claims we prove the statement.

For every $n \geq 2$, let $\left(A_{n}, d_{n}\right)$ be the set $\left\{\left(\frac{i}{n^{2}}, \frac{j}{n^{2}}\right): 0 \leq i, j \leq n\right\}$ equipped with the Euclidean distance $d_{n}$ inherited from $\mathbb{R}^{2}$. Denote by $K$ the amalgamated metric sum of $A_{n}$ 's over 0 . That is, we take $K$ to be the disjoint union $\coprod_{n} A_{n}$ with the zero element $(0,0)$ identified in all of them. The metric $d$ on $K$ is defined as follows. For $a, b \in K$ we set

$$
d(a, b)= \begin{cases}d_{n}(a, b) & \exists n\left(a, b \in A_{n}\right), \\ d_{n}(a, 0)+d_{m}(b, 0) & a \in A_{n}, b \in A_{m}, n \neq m .\end{cases}
$$

It is easy to check that $K$ is a countable compact metric space; in fact, it is a convergent sequence, i.e., it has only one accumulation point - the zero.

Claim 1. $\mathcal{F}(K)$ is isometric to $\bigoplus_{\ell_{1}} \mathcal{F}\left(A_{n}\right)$.

Proof. This is easy and proved e.g. in [17, Proposition 5.1].

For every $n$, consider the set $n A_{n}:=\left\{\left(\frac{i}{n}, \frac{j}{n}\right): 0 \leq i, j \leq n\right\}$ again equipped with the Euclidean distance. Clearly, $\mathcal{F}\left(n A_{n}\right)$ is isometric to $\mathcal{F}\left(A_{n}\right)$. Indeed, since both spaces are finite-dimensional, it suffices to find an isometry of their duals; the mapping $\phi: \operatorname{Lip}_{0}\left(A_{n}\right) \rightarrow \operatorname{Lip}_{0}\left(n A_{n}\right)$ defined by $\phi(f)(x):=n f\left(\frac{x}{n}\right), x \in n A_{n}, f \in \operatorname{Lip}_{0}\left(A_{n}\right)$ is such an isometry. As a consequence we get the following.

Claim 2. $\mathcal{F}(K)$ is linearly isometric to $\bigoplus_{\ell_{1}} \mathcal{F}\left(n A_{n}\right)$.

Since $n A_{n}$, for each $n$, is a subset of $[0,1]^{2}$ we may and will consider $\mathcal{F}\left(n A_{n}\right)$ as a subspace of $\mathcal{F}\left([0,1]^{2}\right)$. Notice that $\bigcup_{n} n A_{n}$ is dense in $[0,1]^{2}$. We need one more technical claim which says that finite dimensional subspaces of $\mathcal{F}\left([0,1]^{2}\right)$ can be approximated by finite dimensional subspaces of $\mathcal{F}\left(n A_{n}\right)$ for large enough $n$. In the following, by $d_{B M}$ we denote the Banach-Mazur distance.

Claim 3. Let $E \subseteq \mathcal{F}\left([0,1]^{2}\right)$ be a finite dimensional subspace and let $\varepsilon>0$ be arbitrary. Then there exist $n \in \mathbb{N}$ and a finite dimensional subspace $E^{\prime} \subseteq \mathcal{F}\left(n A_{n}\right)$ such that $d_{B M}\left(E, E^{\prime}\right)<1+\varepsilon$.

Proof. Let $e_{1}, \ldots, e_{m}$ be a basis of $E$. Since all norms on $E$ are equivalent, there is $D>0$ such that for all $a \in \mathbb{R}^{m}$ we have $\sum_{i=1}^{m}|a(i)| \leq D\left\|\sum_{i=1}^{m} a(i) e_{i}\right\|$. Fix $\delta=\frac{\varepsilon}{2+\varepsilon}$, i.e., such that $\frac{1+\delta}{1-\delta}=1+\varepsilon$. Each $e_{i}$ can be $(\delta / 2 m D)$-approximated by some linear combination of elements from $\operatorname{span}\left\{\delta_{y}: y \in\right.$ $\left.[0,1]^{2}\right\}$. Without loss of generality, we may assume that for each $i \leq m$ such a linear combination is of the same length. So for each $i \leq m$, we choose some $\alpha_{1}^{i} \delta_{x_{1}^{i}}+\ldots+\alpha_{l}^{i} \delta_{x_{l}^{i}} \in \operatorname{span}\left\{\delta_{y}: y \in[0,1]^{2}\right\}$ such that $\left\|e_{i}-\left(\alpha_{1}^{i} \delta_{x_{1}^{i}}+\ldots+\alpha_{l}^{i} \delta_{x_{l}^{i}}\right)\right\|<\delta / 2 m l D$.

Now, since $\bigcup_{n \in \mathbb{N}} n A_{n}$ is dense in $[0,1]^{2}$, if we take $n$ large enough then for every $i \leq m$ and $j \leq l$ we can find $a_{j}^{i} \in n A n$ such that $\left\|\delta_{x_{j}^{i}}-\delta_{a_{j}^{i}}\right\|<\frac{\delta}{2 m l \alpha D}$, where $\alpha=\max \left\{\left|\alpha_{j}^{i}\right|: j \leq l, i \leq m\right\}$. Consequently, for every $i \leq m$ we get

$$
\left\|\alpha_{1}^{i} \delta_{x_{1}^{i}}+\ldots+\alpha_{l}^{i} \delta_{x_{l}^{i}}-\left(\alpha_{1}^{i} \delta_{a_{1}^{i}}+\ldots+\alpha_{l}^{i} \delta_{a_{l}^{i}}\right)\right\|<\alpha \cdot l \cdot \frac{\delta}{2 m l \alpha D}=\delta / 2 m D .
$$


Thus, if for every $i \leq m$ we denote $\alpha_{1}^{i} \delta_{a_{1}^{i}}+\ldots+\alpha_{l}^{i} \delta_{a_{l}^{i}}$ by $e_{i}^{\prime}$, we have $\left\|e_{i}-e_{i}^{\prime}\right\|<\delta / m D$. Hence, for any $a \in \mathbb{R}^{m}$, we have $\left\|\sum_{i=1}^{m} a(i) e_{i}-\sum_{i=1}^{m} a(i) e_{i}^{\prime}\right\|<\delta / D\left(\sum_{i=1}^{m}|a(i)|\right) \leq \delta\left\|\sum_{i=1}^{m} a(i) e_{i}\right\|$ and, consequently,

$$
\left\|\sum_{i=1}^{m} a(i) e_{i}^{\prime}\right\|<(1+\delta)\left\|\sum_{i=1}^{m} a(i) e_{i}\right\| \quad \text { and } \quad\left\|\sum_{i=1}^{m} a(i) e_{i}\right\|<\left\|\sum_{i=1}^{m} a(i) e_{i}^{\prime}\right\|+\delta\left\|\sum_{i=1}^{m} a(i) e_{i}\right\| .
$$

Denote by $E^{\prime}$ the subspace $\operatorname{span}\left\{e_{i}^{\prime}: i \leq m\right\} \subseteq \mathcal{F}\left(n A_{n}\right)$. Using the above, the linear mapping determined by sending $e_{i}$ to $e_{i}^{\prime}$, for $i \leq m$, is a witness of the fact that $d_{B M}\left(E, E^{\prime}\right)<\frac{1+\delta}{1-\delta}=1+\varepsilon$.

Let us now formulate a result of Lindenstrauss and Pełczyński that will help us finish the proof.

Theorem 9 (Theorem 7.1 in [21]). Let $X$ be a Banach space and fix $\lambda \geq 1$. If for every finite dimensional subspace $E$ of $X$ there exists a finite dimensional subspace $E^{\prime}$ of $\ell_{1}$ such that $d_{B M}\left(E, E^{\prime}\right) \leq \lambda$, then there exists a measure $\mu$ and a subspace $Y$ of $L_{1}(\mu)$ such that $d_{B M}(X, Y) \leq \lambda$.

We are now ready to finish the proof of Theorem 2 . By Theorem 7 we have that $\mathcal{F}\left([0,1]^{2}\right)$ does not embed into $L_{1}(\mu)$ for any measure $\mu$. However, then by Theorem 9 we get that for every $N \in \mathbb{N}$ there exists a finite dimensional subspace $E_{N}$ of $\mathcal{F}\left([0,1]^{2}\right)$ such that for every finite dimensional subspace $F$ of $\ell_{1}$ we have $d_{B M}\left(E_{N}, F\right)>N$. Using Claim 3, for each $N$ we can find some $n(N) \in \mathbb{N}$ and finite dimensional subspace $E_{n(N)}$ of $\mathcal{F}\left(n(N) A_{n(N)}\right)$ such that $d_{B M}\left(E_{N}, E_{n(N)}\right)<2$.

Assume now that $\mathcal{F}(K)$ embeds into $L_{1}$ via some linear embedding of norm less than $N / 8$ for some $N \in \mathbb{N}$. By Claim 2, $\oplus_{\ell_{1}} \mathcal{F}\left(n A_{n}\right)$ embeds into $L_{1}$ via some linear embedding $T$ of norm less than $N / 8$. Now, $T$ restricted on $E_{n(N)} \subseteq \mathcal{F}\left(n(N) A_{n(N)}\right)$ has still norm bounded by $N / 8$. In particular, there is some finite dimensional subspace $Y_{n(N)}$ of $L_{1}$ such that $d_{B M}\left(E_{n(N)}, Y_{n(N)}\right) \leq N / 8$. Since $L_{1}$ is finitely representable in $\ell_{1}$, see [2, Proposition 11.1.7], there exists a finite dimensional subspace $Y_{N}$ of $\ell_{1}$ such that $d_{B M}\left(Y_{N}, Y_{n(N)}\right)<2$.

Now putting all these inequalities together we get

$$
\frac{N}{8} \geq d_{B M}\left(E_{n(N)}, Y_{n(N)}\right) \geq \frac{d_{B M}\left(E_{N}, Y_{N}\right)}{d_{B M}\left(E_{N}, E_{n(N)}\right) \cdot d_{B M}\left(Y_{N}, Y_{n(N)}\right)}>\frac{N}{4}
$$

and that is a contradiction finishing the proof.

Remark 10. The paper [17] was published (see [18]). However, in the published version the statement [17, Proposition 5.1], which we cite in the proof of Claim 1 above is missing. Thus, we would like to sketch the easy proof of it here.

Consider

$$
\Phi: \bigoplus_{\ell_{\infty}} \operatorname{Lip}_{0}\left(A_{n}\right) \rightarrow \operatorname{Lip}_{0}(K)
$$

defined by $\Phi\left(\left(f_{n}\right)\right)(x)=f_{n}(x),\left(f_{n}\right) \in \bigoplus_{\ell_{\infty}} \operatorname{Lip}_{0}\left(A_{n}\right), x \in A_{n}$. Then it is easy to verify that $\Phi$ is an isometry onto and $w^{*}-w^{*}$ homeomorphism. Hence, it is the adjoint of an isometry from $\mathcal{F}(K)$ onto $\bigoplus_{\ell_{1}} \mathcal{F}\left(A_{n}\right)$.

Remark 11. It has been observed by G. Lancien and A. Procházka that our method of proof actually gives that $K$ from the statement of Theorem 2 can be taken as a subset of $[0,1]^{2}$ and that there does not exist a bi-Lipschitz embedding of $\mathcal{F}(K)$ into $L_{1}$. Let us sketch the argument here.

First, the only place where we used the metric of $K$ was to prove Claim 1. However, it is easy to see that taking a sequence $\left(k_{n}\right)_{n \in \mathbb{N}}$ increasing fast enough, we have that $\mathcal{F}\left(\bigcup_{n \in \mathbb{N}} A_{k_{n}}\right)$ is linearly isomorphic to $\bigoplus_{\ell_{1}} \mathcal{F}\left(A_{k_{n}}\right)$ (using the same mapping $\Phi$ as in Remark 10), which would be enough for the rest of the proof. Hence, we may have $K=\bigcup_{n \in \mathbb{N}} A_{k_{n}}$. Moreover, our proof gives that $\mathcal{F}(K)$ does not linearly embed into any Banach space finitely representable in $\ell_{1}$. If there was a 
bi-Lipschitz embedding of $\mathcal{F}(K)$ into $L_{1}$ then, by [3, Corollary 7.10], $\mathcal{F}(K)$ embeds linearly into $\left(L_{1}\right)^{* *}$ which is by the principle of local reflexivity [2, Theorem 11.2.4] finitely represented in $L_{1}$ (in particular, $\left(L_{1}\right)^{* *}$ is finitely represented in $\ell_{1}$ because $L_{1}$ is), a contradiction.

\section{Embedding of $c_{0}$}

Let $M$ be a separable metric space that contains a bi-Lipschitz copy of every separable metric space. By [14, Theorems 2.12 and 3.1], we have $X \hookrightarrow \mathcal{F}(X)$ for every separable Banach space $X$; therefore, $\mathcal{F}(M)$ is a universal separable Banach space, i.e., $\mathcal{F}(M)$ contains an isomorphic copy of every separable Banach space. Note that by the result of Aharoni [1] this is equivalent to the condition that $M$ contains a bi-Lipschitz copy of $c_{0}$. Y. Dutrieux and V. Ferenczi in [9] asked for the converse, see Question 1. In this section we prove Theorem 3, making a partial progress towards the answer to this question.

Let $M$ be either $[0,1]^{n}$ or $\mathbb{R}^{n}$. By $\mathcal{C}^{1}(M)$ we denote the space of functions $F: M \rightarrow \mathbb{R}$ whose derivatives of order $\leq 1$ are continuous on $M$. For $F \in \mathcal{C}^{1}(M)$ we define $\|F\|_{\infty}^{1}:=\max \left\{\|F\|_{\infty},\left\|\partial_{x_{i}} F\right\|_{\infty}\right.$ : $i \leq n\}$. It is well-known that the space $\left(\mathcal{C}^{1}\left([0,1]^{n}\right),\|\cdot\|_{\infty}^{1}\right)$ is a Banach space.

The following result was essentially proved by J. Bourgain [5], [6]. The result of J. Bourgain concerns the space of smooth functions over $n$-dimensional torus; however, the same proof works for the $n$-dimensional cube. We refer also to [28], where a more detailed proof of the result of J. Bourgain may be found (use Example III.D.30 and Theorem III.D.31 and conclude similarly as in the proof of Corollary III.C.14).

Theorem 12. For every $n \in \mathbb{N}$, the Banach space $\left(\mathcal{C}^{1}\left([0,1]^{n}\right)\right)^{*}$ is weakly sequentially complete, i.e., weakly Cauchy sequences are weakly convergent.

Lemma 13. Let $A \subset \mathbb{R}^{n}$ be a finite set and $f: A \rightarrow \mathbb{R}$ a 1-Lipschitz function (on $\mathbb{R}^{n}$ we consider Euclidean norm). Then, for every $\varepsilon>0$, there exists $g \in \mathcal{C}^{1}\left(\mathbb{R}^{n}\right)$, an extension of $f$ (i.e., $g \supset f$ ), with $\|g\|_{\infty}^{1}<\max \left\{\|f\|_{\infty}, 1\right\}+\varepsilon$.

Proof. Find $\delta>0$ such that the balls $\{B(a, 2 \delta): a \in A\}$ are pairwise disjoint. Fix some even Lipschitz function $\tau \in \mathcal{C}^{1}(\mathbb{R})$ with $\tau(0)=1,\|\tau\|_{\infty} \leq 1$ and $\{x: \tau(x) \neq 0\} \subset(-\delta, \delta)$; e.g.

$$
\tau(x)= \begin{cases}e^{-\frac{1}{\delta^{2}-x^{2}}+\frac{1}{\delta^{2}}} & |x|<\delta, \\ 0 & \text { otherwise. }\end{cases}
$$

Let $K$ be such that $\tau$ is $K$-Lipschitz and $K>1$.

We may assume that $0 \in A$ and $f(0)=0$. First, we extend $f$ to a 1-Lipchitz function defined on $\mathbb{R}^{n}$; see e.g. [16, Lemma 7.39]. We call this extension again $f$. Now, we find a 1-Lipschitz $\tilde{g} \in \mathcal{C}^{1}\left(\mathbb{R}^{n}\right)$ with $\|f-\tilde{g}\|_{\infty}<\varepsilon / 2 K$; e.g. using the standard integral convolution [16, Lemma 7.1].

For $a \in A$ define $\phi_{a}: \mathbb{R}^{n} \rightarrow \mathbb{R}$ by $\phi_{a}(x)=(f(a)-\tilde{g}(a)) \tau(\|x-a\|), x \in \mathbb{R}^{n}$. Then $h:=\sum_{a \in A} \phi_{a}$ is a well-defined $\varepsilon / 2$-Lipschitz function such that $\|h\|_{\infty} \leq \varepsilon / 2 K$ and $h(a)=f(a)-\tilde{g}(a)$ for every $a \in A$. Moreover, since on a Hilbert space the norm is smooth everywhere except 0 and since the function $\tau$ is even, it is easy to observe that $h \in \mathcal{C}^{1}\left(\mathbb{R}^{n}\right)$. It remains to put $g:=\tilde{g}+h$. Then we have $g \in \mathcal{C}^{1}\left(\mathbb{R}^{n}\right),\|g\|_{\infty}<\|f\|_{\infty}+\varepsilon$ and $g$ is $(1+\varepsilon / 2)$-Lipschitz; hence, $\|g\|_{\infty}^{1}<\max \left\{\|f\|_{\infty}, 1\right\}+\varepsilon$.

Remark 14. Note that it follows from Lemma 13 that whenever $A$ is a finite set in $[0,1]^{n}$ and $f$ a 1-Lipschitz function on $A$ with $f(0)=0$, there is $g \in \mathcal{C}^{1}\left(\mathbb{R}^{n}\right)$ with $\|g\|_{\infty}^{1} \leq \sqrt{n}+1$. Therefore, by [10, Theorem 1], there is a linear extension operator $T: \operatorname{Lip}_{0}(A) \rightarrow \operatorname{Lip}_{0}\left(\mathbb{R}^{n}\right)$ with norm depending only on $n$; hence, $\left.T^{*}\right|_{\mathcal{F}\left(\mathbb{R}^{n}\right)}$ is a projection from $\mathcal{F}\left(\mathbb{R}^{n}\right)$ onto $\mathcal{F}(A)$. Consequently, whenever we have $M \subset[0,1]^{n}$ and $A \subset M$ a finite set, $\mathcal{F}(A)$ is $C(n)$-complemented in $\mathcal{F}(M)$, where the constant $C(n)$ depends only on the dimension $n$. This gives another proof of the fact that $\mathcal{F}(M)$ has BAP whenever $M \subset[0,1]^{n}[20$, Proposition 2.3]. 
Lemma 15. For every $n \in \mathbb{N}$, there is an isomorphism of $\mathcal{F}\left([0,1]^{n}\right)$ into $\left(\mathcal{C}^{1}\left([0,1]^{n}\right)\right)^{*}$.

Proof. Put $Y=\left\{f \in \mathcal{C}^{1}\left([0,1]^{n}\right): f(0)=0\right\}$. Then $Y$ is a closed subspace of codimension 1 ; hence, it is complemented and $Y^{*}$ is isomorphic to a subspace of $\left(\mathcal{C}^{1}\left([0,1]^{n}\right)\right)^{*}$. For every $x \in[0,1]^{n}$ we define $T\left(\delta_{x}\right) \in Y^{*}$ by $T\left(\delta_{x}\right)(f):=f(x), f \in Y$. Extend $T$ linearly to the set $\operatorname{span}\left\{\delta_{x}: x \in[0,1]^{n}\right\}$. Now, it is enough to verify that $T$ is an isomorphism into $Y^{*}$.

Fix an element $\mu \in \operatorname{span}\left\{\delta_{x}: x \in[0,1]^{n}\right\}$. There are $k \in \mathbb{N}, \alpha \in \mathbb{R}^{k}$ and $x_{1} \ldots, x_{k} \in[0,1]^{n}$ with $\mu=\sum_{i=1}^{k} \alpha(i) \delta_{x_{i}}$. We have to find constants $C>0$ and $D>0$ with

$$
\begin{aligned}
C \sup \left\{\left|\sum_{i=1}^{k} \alpha(i) f\left(x_{i}\right)\right|:\|f\|_{\infty}^{1} \leq 1, f(0)=0\right\} & \leq \sup \left\{\left|\sum_{i=1}^{k} \alpha(i) f\left(x_{i}\right)\right|:\|f\|_{\text {Lip }} \leq 1, f(0)=0\right\} \\
& \leq D \sup \left\{\left|\sum_{i=1}^{k} \alpha(i) f\left(x_{i}\right)\right|:\|f\|_{\infty}^{1} \leq 1, f(0)=0\right\} .
\end{aligned}
$$

The existence of constant $C$ follows from the basic fact that every function with total differential bounded by $K$ is $K$-Lipschitz; see [26, Theorem 9.19]; hence, we may put $C=1 / \sqrt{n}$. The existence of $D$ follows from Lemma 13, which gives $D=\sqrt{n}$.

Proof of Theorem [3. By [18, Corollary 3.3], $\mathcal{F}\left(\mathbb{R}^{n}\right)$ is isomorphic to $\mathcal{F}\left([0,1]^{n}\right)$. Hence, $\mathcal{F}\left(\mathbb{R}^{n}\right)$ is weakly sequentially complete by Theorem 12 and Lemma 15. Finally, using the fact mentioned in Section 1 that $\mathcal{F}(M)$ is isometric to a subspace of $\mathcal{F}\left(\mathbb{R}^{n}\right)$, we see that $\mathcal{F}(M)$ is weakly sequentially complete. Consequently, $c_{0}$ does not embed isomorphically into $\mathcal{F}(M)$ because, as it is well known and easy to prove, $c_{0}$ is not weakly sequentially complete.

\section{Open PROBlems}

As it was mentioned in Section 4, if $M$ contains a bi-Lipschitz copy of $c_{0}$, then $\mathcal{F}(M)$ is a universal separable Banach space. Hence, we have quite a rich family of universal separable Banach spaces. By Theorem 1, they are all different from $\mathcal{C}(K)$ spaces and from the Gurariu space. One example is Pełczyński's universal basis space $\mathbb{P}$ (which is unique up to isomorphism). This space is isomorphic to $\mathcal{F}(\mathbb{P})$, see [14, p. 139]. Another example is the Holmes space, i.e., the Lipschitz-free space over the Urysohn universal metric space. By [11, Theorem 4.2], the Holmes space is not isomorphic to $\mathbb{P}$. By [9, Theorem 5], $\mathcal{F}\left(c_{0}\right)$ is isomorphic to each $\mathcal{F}(\mathcal{C}(K))$. It could be of some interest to find out what isomorphic types of universal Banach spaces we are able to get using the Lipschitz-free construction. For example, the following seems to be open.

Question 2. Is $\mathcal{F}\left(c_{0}\right)$ isomorphic to the Holmes space or to $\mathbb{P}$ ?

In the light of Theorem 3 it is also natural to ask the following.

Question 3. Is it true that $c_{0} \hookrightarrow \mathcal{F}\left(\ell_{2}\right)$ ?

Note that $c_{0}$ does not bi-Lipschitz embed into $\ell_{p}(1 \leq p<\infty)$, see e.g. [3, p. 169]. Hence, the negative answer to the above question would be a partial progress towards the answer to Question 1. Similarly, we do not know the answer to the following question.

Question 4. Is it true that $c_{0} \hookrightarrow \mathcal{F}\left(\ell_{1}\right)$ ?

Acknowledgements. We wish to thank G. Lancien and A. Procházka for the valuable observation contained in Remark 11. M. Cúth was supported by Warsaw Center of Matemathics and Computer Science (KNOW-MNSzW). M. Doucha was supported by funds allocated to the implementation of the international co-funded project in the years 2014-2018, 3038/7.PR/2014/2, and by the EU grant PCOFUND-GA-2012-600415. P. Wojtaszczyk was supported by Polish NCN grant UMO2011/03/B/ST1/04902. 


\section{REFERENCES}

[1] I. Aharoni, Every separable metric space is Lipschitz equivalent to a subset of c o $^{+}$, Israel J. Math., 19 (1974), pp. 284-291.

[2] F. Albiac And N. J. Kalton, Topics in Banach space theory, vol. 233 of Graduate Texts in Mathematics, Springer, New York, 2006.

[3] Y. Benyamini and J. Lindenstrauss, Geometric nonlinear functional analysis. Vol. 1, vol. 48 of American Mathematical Society Colloquium Publications, American Mathematical Society, Providence, RI, 2000.

[4] C. Bessaga And A. Pęczyński, On bases and unconditional convergence of series in Banach spaces, Studia Math., 17 (1958), pp. 151-164.

[5] J. Bourgain, On weak completeness of the dual of spaces of analytic and smooth functions, Bull. Soc. Math. Belg. Sér. B, 35 (1983), pp. 111-118.

[6] — The Dunford-Pettis property for the ball-algebras, the polydisc-algebras and the Sobolev spaces, Studia Math., 77 (1984), pp. 245-253.

[7] M. Cúth And M. Doucha, Lipschitz-free spaces over ultrametric spaces, Mediterr. J. Math. electronically published on May 03, 2015, DOI: http://dx.doi.org/10.1007/s00009-015-0566-7 (to appear in print).

[8] A. Dalet, Free spaces over countable compact metric spaces, Proc. Amer. Math. Soc. electronically published on February 25, 2015, DOI: http://dx.doi.org/10.1090/S0002-9939-2015-12518-X (to appear in print).

[9] Y. Dutrieux and V. Ferenczi, The Lipschitz free Banach spaces of $C(K)$-spaces, Proc. Amer. Math. Soc., 134 (2006), pp. 1039-1044 (electronic).

[10] C. Fefferman, Interpolation and extrapolation of smooth functions by linear operators, Rev. Mat. Iberoamericana, 21 (2005), pp. 313-348.

[11] V. P. Fonf and P. Wojtaszczyk, Properties of the Holmes space, Topology Appl., 155 (2008), pp. $1627-1633$.

[12] J. Garbulińska And W. Kubiś, Remarks on Gurariu spaces, Extracta Math., 26 (2011), pp. $235-269$.

[13] A. Godard, Tree metrics and their Lipschitz-free spaces, Proc. Amer. Math. Soc., 138 (2010), pp. 4311-4320.

[14] G. Godefroy and N. J. Kalton, Lipschitz-free Banach spaces, Studia Math., 159 (2003), pp. 121-141. Dedicated to Professor Aleksander Pełczyński on the occasion of his 70th birthday.

[15] V. I. GURARĬ, Spaces of universal placement, isotropic spaces and a problem of Mazur on rotations of Banach spaces, Sibirsk. Mat. Ž., 7 (1966), pp. 1002-1013.

[16] P. HÁJeK And M. Johanis, Smooth analysis in Banach spaces, vol. 19 of De Gruyter Series in Nonlinear Analysis and Applications, De Gruyter, Berlin, 2014.

[17] P. Kaufmann, Products of lipschitz-free spaces and applications, (2014). preprint avaiable at http://arxiv.org/pdf/1403.6605.pdf

[18] P. L. Kaufmann, Products of Lipschitz-free spaces and applications, Studia Math., 226 (2015), pp. $213-227$.

[19] S. V. Kisluakov, Sobolev imbedding operators, and the nonisomorphism of certain Banach spaces, Funkcional. Anal. i Priložen., 9 (1975), pp. 22-27.

[20] G. LANCIEn AND E. PERnECKÁ, Approximation properties and Schauder decompositions in Lipschitz-free spaces, J. Funct. Anal., 264 (2013), pp. 2323-2334.

[21] J. Lindenstrauss And A. PeŁczý́ski, Absolutely summing operators in $L_{p}$-spaces and their applications, Studia Math., 29 (1968), pp. 275-326.

[22] A. Naor And G. Schechtman, Planar earthmover is not in L $L_{1}$, SIAM J. Comput., 37 (2007), pp. 804-826 (electronic).

[23] A. Nekvinda And L. Zajíček, A simple proof of the Rademacher theorem, Časopis Pěst. Mat., 113 (1988), pp. 337-341.

[24] I. Protasov, Combinatorics of numbers, vol. 2 of Mathematical Studies Monograph Series, VNTL Publishers, L'viv, 1997. Translated from the Russian manuscript by Michael Zarichnyi and Taras Banakh.

[25] H. P. Rosenthal, The Banach spaces $C(K)$, in Handbook of the geometry of Banach spaces, Vol. 2, NorthHolland, Amsterdam, 2003, pp. 1547-1602.

[26] W. Rudin, Principles of mathematical analysis, McGraw-Hill Book Co., New York-Auckland-Düsseldorf, third ed., 1976. International Series in Pure and Applied Mathematics.

[27] N. Weaver, Lipschitz algebras, World Scientific Publishing Co., Inc., River Edge, NJ, 1999.

[28] P. Wojtaszczyk, Banach spaces for analysts, vol. 25 of Cambridge Studies in Advanced Mathematics, Cambridge University Press, Cambridge, 1991.

E-mail address: marek.cuth@gmail.com, m.doucha@post.cz, wojtaszczyk@icm.edu.pl

(M. Cúth) Charles University, Faculty of Mathematics and Physics, Department of Mathematical Analysis, Sokolovská 83, 18675 Prague 8, Czech Republic 
(M. Cúth, M. Doucha) Instytut Matematyczny Polskiej Akademi Nauk, Śniadeckich 8, 00-656 Warszawa, POLAND

(P. Wojtaszczyk) Interdisciplinary Centre for Mathematical and Computational Modelling, UniverSity of Warsaw, ul. Prosta 69, 02-838 Warszawa, Poland 